\title{
The Pathogenesis of Multiple Sclerosis
}

\section{Gejalakshmi S* and Harikrishnan N}

Faculty of Pharmacy, Dr. M.G.R. Educational and Research Institute, Chennai, India *Corresponding Author: Gejalakshmi S, Faculty of Pharmacy, Dr. M.G.R. Educational and Research Institute, Chennai, India.
Received: March 14, 2020;

Published: April 10, 2020

(C) All rights are reserved by Gejalakshmi S and Harikrishnan $\mathbf{N}$.

\begin{abstract}
An array of recently published manuscript globally have proposed the stages of different pathogenic pathways in multiple sclerosis. These research were focused on both biopsy and autopsy. Based the review data on clinical, imaging and cerebrospinal fluid suggest that most of the cases were disseminated encephalomyelitis rather than MS pathogenesis. Cognitive impairment is a common and disabling feature of multiple sclerosis. It may contribute to withdrawal from work than physical disability. The article delivers the importance for early diagnosis and management of disabilities in MS, before it develops an irreversible entry. Keywords: Multiple Sclerosis; Autopsy; Biopsy; Encephalomyelitis; Physical Disability
\end{abstract}

\section{Introduction}

Over the past few decades enormous articles drafted by a group of neuropathologist have provided an novel approach in the pathogenesis of multiple sclerosis (MS). These new ideas were highly impact which leads to innovative therapeutic guideline for treating MS. By the same concept, differentiating MS from other inflammatory diseases, both clinically and in terms of diseases assumes great importance. It has observed that T-cell mediated immune response is the commonest source in the development of MS lesions. Antibodies migrated against surface membrane of myelin sheet are one of the factor involved in demyelination process [1]. Multiple sclerosis (MS) is an central nervous system (CNS) disorder. It is an autoimmune origin in which $\mathrm{T}$ cells specifically recognizing myelin fragments induce tissue damage and leads to lesion in the CNS. The inflammatory response leads to demyelination and axonal and neuronal loss. Various symptoms of MS include numbness, paresis, visual disturbance, bladder dysfunction and others. The disease to be mainly on the white matter of the CNS. Over last years affects grey matter. Prevalence of MS differ depending on the region of the world with most affected patients distant from the equator [2]. The genetic influence is mainly mediated by HLA II genes. Environmental influences are demonstrated by migration studies. There is an influence of the vitamin D level and sun exposure with reduction of susceptibility with increased serum vitamin D levels It has been demonstrated that also smoking has a strong negative influence on MS in context of certain HLA genes. Various multiple events leading to MS [3].

\section{Clinical manifestation of MS [4]}

There are different types of MS. Nearly $85 \%$ of MS patients initially present with a relapsing-remitting type of MS. In certain cases there is a conversion over time to a secondary chronic progressive type of MS (SP-MS). About $15 \%$ of cases present with a primary progressive disease course (PP-MS). A small percentage of these patients has a progressive relapsing MS disease course (PR-MS) with fast progressive type of disease [5,6]. A relapse can be diagnosed if a patient has new symptoms or an aggravation of symptoms that lasts for more than $24 \mathrm{~h}$. To diagnose MS, the appearance of symptoms and the presence of lesions in space and time in the CNS as demonstrated by nuclear magnetic resonance (NMR) is needed.

Pathology of lesions in MS [6]

Ms lesions are classified on the basis of myelin laden macrophages. In acute and chronic active lesions the axons are preserved while any macrophages present in these lesions that have taken up myelin. In contrast, in inactive plaques there is loss of axons and oligodendrocytes and only few macrophages or/and microglia cells are present [7]. There are also smoldering plaques that represent an intermediate stage of chronic active and inactive lesions with a hypocellular center and the presence of macrophages at the sharp borders. Shadow plaques represent remyelinated lesions.

Immune response in MS-key players of innate and adaptive immunity

The immune system is comprised of the innate and the adaptive immune response. Insights into both systems have their roles 
in humans have come from immune defects While components of the innate immune response are reacting very fast to changes in the homeostasis, the adaptive immune response takes more time but reacts in a much more specific fashion $[8,9]$.

Influence of the systemic immune response on MS

Most MS researchers believe that the systemic immunity is of major importance in MS. Possibly MS is induced as an unwanted side effect of a systemic immune response against foreign 'nonself' antigens $[10,11]$. This could be due to molecular mimicry or bystander activation. Molecular mimicry indicates that 'non-self' derived antigens share structural homology to 'self' antigens and that this homology can lead to induction of CNS autoimmunity [12$15]$.

Basis of disease initiation: The pathologic lesions that corelates with clinical conditions of disease which focus on inflammation associated with active myelin degradation and phagocytosis [16-18]. A present research is to identify whether this neuro inflammatory reaction is based on immune system or in response to primary events impacting on the neural cells.

\section{Causal factors in MS - general considerations}

Chronic diseases such as MS typically have complex etiological bases, which generally involve an interaction between the individual's genetic background and the environmental events that they experience during their lives. For example, as noted earlier, an individual from northern North America or northern Europe has a life-time risk of developing MS of approximately $0.1-0.2 \%$ $[19,20]$. The risk for an individual with an affected family member increases roughly in proportion to the genetic similarity between themselves and the proband. Siblings of an MS proband $(\sim 50 \%$ genetic similarity) have a 20 - 30 fold increased risk compared to the general population. By contrast, monozygotic-twins $(\sim 100 \%$ genetic similarity) have an MS risk that is approximately 10 times this sibling risk. Despite this evidence of a strong genetic predisposition, however, genetics is clearly not the only determinant. If it were, the proband-wise concordance-rate for monotonic-twins should be much closer to $100 \%$ than the reported $25-30 \%$ in the highest-prevalence populations. In populations with lower prevalence, where the concordance-rate for monozygotic-twins is one quarter to one half that in the north, this conclusion is even more evident. As a result, in addition to genetic determinants, there clearly must also be environmental and/or epigenetic factors that contribute in important ways to MS pathogenesis [21].

\section{Novel approaches for discovery of underlying pathology in MS}

Genetics has helped to elucidate some underlying mechanisms of MS and has put antigen presentation and the T cell in the center of the MS pathology [22]. A major field that will further help to understand MS is epigenetics. As data is emerging that underscores the importance of epigenetics. Hence incomplete understanding of the mechanisms that drive chronicity and neural degeneration.
Possibly a lot will be learned by technologies such as two photon microscopy [23], imaging of cellular compartments and axonal transport. good tool to study aspects of MS immunopathogenesis and neurobiology [24]. When using EAE it should always be clear that one. Humanization is of great importance in modeling, nevertheless it must be acknowledged that the degree of humanization of models is low and the predictability of results obtained in humanized animal models compared to wild type animal models is not necessarily higher [25]. Possibly, the use of marmosets with their higher genetic proximity to humans compared to rodents offers some advantages for specific well defined questions of great importance Neuromyelitis optica (NMO) is a special disease entity that is supposed to be caused in most cases by antibodies against aquaporin-4. Aquaporin-4 is a water channel expressed on astrocytes [26]. In order to diagnose NMO, patients need to present with optic neuritis and acute myelitis. In addition, there should be two of three supportive criteria which include firstly a negative brain MRI at onset, secondly a spinal cord MRI with contiguous T2 weighted signal abnormality extending over 3 or more vertebral segments and thirdly seropositivity for acquaporin-4 antibodies. So far it is not completely solved if NMO is a subtype of MS or a completely separate disease entity. NMO is presently treated by corticosteroids, azathioprine, mycophenolate mofetil, mitoxantrone, cyclophosphamide, intravenous immune globulin (IVIG), rituximab, plasmapheresis or eculizumab a mAb directed against the complement factor C5 So far no large controlled trials have been conducted, although at present much effort is being done to find efficacious therapies [27]. So far rituximab treatment that leads to B cell depletion seems to have the best clinical outcomes. Classical MS therapies like interferon-beta preparations, natalizumab and fingolimod have not worked or rather have increased disease severity. Therefore, much care should be taken in the diagnosis and treatment of NMO.

\section{Causal environmental factors in MS}

Over the years, many environmental factors, including physical trauma, psychological stress, vaccinations, smoking tobacco, typhoid, small pox, Epstein-Barr virus (EBV), human herpes virus (HHV), chicken pox, Chlamydia, other infections, vitamin deficiencies, low-sunlight, cosmic-rays, ionizing radiation, occupational hazards, living with domesticated animals, dietary habits, mercury amalgam tooth fillings and toxic exposures have all been postulated to be linked to MS pathogenesis [28]. Although the evidence for many of these factors is quite weak, some continue to have strong proponents and no single factor has been established as associated. Neither can any factor be excluded completely although many lack credible scientific evidence, biological plausibility, or both. Consequently, here the focus will be on the potential relationship of MS to Vitamin D deficiency and/or EBV infection [29].

\section{Interactions between genetics and the environment}

Interaction has been observed between genetic make up and an environment which are indicated by the observation that the proband-wise monozygotic-twin concordance-rate for women it is 
significantly larger the same rate for men. Results of consequences of observed difference proves that current prevalence of MS is greater in women than men For example, considering some of the potential environmental factors, it might be that women use more sun-block or sun-avoidance than men and, therefore, they experience greater vitamin D deficiency [30]. Or it might be women have better hygiene as children than men and, thus, acquire EBV infections later in life. Alternatively, it could be that there are genderspecific differences in vitamin D metabolism, such that men and women experience a deficiency at different levels of absolute exposure [31]. Also, it could be that women have a greater likelihood of actually developing MS once the necessary environmental and genetic events have come together or it could be that some combination of these factors contributes to the observed gender-specific differences [32].

\section{Summary and Outlook}

A thorough study of MS has been surveyed. It is very evident that $\mathrm{T}$ cells are in the center of the immune pathogenesis of relapsing MS. This is underscored by the positive response of therapeutic agents that target or modulate $\mathrm{T}$ cells. MS induced cause is unknown. unfamiliar regarding the events that lead to chronic disease unrelated to relapses. Possibly the antigens driving this type of disease are different. other mechanisms might be operative such as dysregulation of ion channels and disturbance of axonal transport due to cellular disturbances or underlying defects.MS is an exciting field to work in and there is still ample opportunity to discover novel pathways leading to tissue damage. In order to do so it is of major importance to work with the best suited models and tools.

\section{Conflict of Interest}

The authors have no conflict of interest.

\section{Bibliography}

1. Till C., et al. "A feasibility study of working memory training for individuals with paediatric-onset multiple sclerosis". Neuropsychological Rehabilitation 29.8 (2019): 1177-1192.

2. Speer G. "Impact of vitamin D in neurological diseases and neurorehabilitation: from dementia to multiple sclerosis. Part I: the role of vitamin D in the prevention and treatment of multiple sclerosis]". Ideggyogy Sz 66.9-10 (2013): 293-303.

3. Ganji A., et al. "Therapeutic effects of walnut oil on the animal model of multiple sclerosis". Nutritional Neuroscience 22.3 (2019): 215-222.

4. Waldman A., et al. "Pediatric multiple sclerosis: Clinical features and outcome". Neurology 87.9-2 (2016): S74-S81.

5. Kozhieva M., et al. "Primary progressive multiple sclerosis in a Russian cohort: relationship with gut bacterial diversity". BMC Microbiology 19.1 (2019): 309.
6. Zeis T., et al. "Molecular pathology of Multiple Sclerosis lesions reveals a heterogeneous expression pattern of genes involved in oligodendrogliogenesis". Experimental Neurology 305 (2018): 76-88.

7. Giorgio A and De Stefano N. "Effective Utilization of MRI in the Diagnosis and Management of Multiple Sclerosis". Neurologic Clinics 36.1 (2018): 27-34.

8. Voet S., et al. "Microglia in Central Nervous System Inflammation and Multiple Sclerosis Pathology". Trends in Molecular Medicine 25.2 (2019): 112-123.

9. Voller LM., et al. "Progressive and translucent plaques on the soles". Cutis 102.5 (2018): 310.

10. Wu J., et al. "Germline mutations of renal cancer predisposition genes and clinical relevance in Chinese patients with sporadic, early-onset disease". Cancer 125.7 (2019): 1060-1069.

11. Xie Y., et al. "Effects of moderate- versus high- intensity swimming training on inflammatory and CD4(+) T cell subset profiles in experimental autoimmune encephalomyelitis mice". Journal of Neuroimmunology 328 (2019): 60-67.

12. Yamout BI., et al. "Safety and Efficacy of Rituximab in Multiple Sclerosis: A Retrospective Observational Study". Journal of Immunology Research (2018): 9084759.

13. Yu FF., et al. "Characterization of normal-appearing white matter in multiple sclerosis using quantitative susceptibility mapping in conjunction with diffusion tensor imaging". Neuroradiology 61.1 (2019): 71-79.

14. Charvet B., et al. "Induction of Proinflammatory Multiple Sclerosis-Associated Retrovirus Envelope Protein by Human Herpesvirus-6A and CD46 Receptor Engagement". Frontiers in Immunology 9 (2018): 2803.

15. Cheng Y., et al. "Innate Immune Responses and Viral-Induced Neurologic Disease”. Journal of Clinical Medicine 8.1 (2018).

16. Costa C., et al. "Expression of Bone Morphogenetic Proteins in Multiple Sclerosis Lesions". The American Journal of Pathology 189.3 (2019): 665-676.

17. Demir S., et al. "Immune cell derived BDNF does not mediate neuroprotection of the murine anti-CD52 antibody in a chronic autoimmune mouse model". Journal of Neuroimmunology 328 (2019): 78-85.

18. Di Pauli F and Berger T. "Myelin Oligodendrocyte Glycoprotein Antibody-Associated Disorders: Toward a New Spectrum of Inflammatory Demyelinating CNS Disorders?" Frontiers in Immunology 9 (2018): 2753. 
19. Kira JI., et al. "Anti-neurofascin autoantibody and demyelination". Neurochemistry International 130 (2019): 104360.

20. Kohler C., et al. "Exploring individual multiple sclerosis lesion volume change over time: Development of an algorithm for the analyses of longitudinal quantitative MRI measures". NeuroImage Clinical 21 (2019): 101623.

21. Giarola B., et al. "Autoimmune encephalitis following alemtuzumab treatment of multiple sclerosis". Multiple Sclerosis and Related Disorders 28 (2019): 31-33.

22. Lassmann H. "Pathogenic Mechanisms Associated With Different Clinical Courses of Multiple Sclerosis". Frontiers in Immunology 9 (2018): 3116.

23. McEneaney LJ and Tee AR. "Finding a cure for tuberous sclerosis complex: From genetics through to targeted drug therapies". Advances in Genetics 103 (2019): 91-118.

24. Omerhodzic I., et al. "Relapsing Tumefactive Demyelination: A Case Report". Acta Medica Academica 47.2 (2018):193-198.

25. Rigopoulou EI., et al. "Autoimmune hepatitis in patients with multiple sclerosis: The role of immunomodulatory treatment". Clinics and Research in Hepatology and Gastroenterology 43.3 (2019): e25-e32.

26. Thompson KK., et al. "Tuftsin Combines With Remyelinating Therapy and Improves Outcomes in Models of CNS Demyelinating Disease". Frontiers in Immunology 9 (2018): 2784.

27. Toader LE., et al. "Clinical and Histopathological Assessment on an Animal Model with Experimental Autoimmune Encephalomyelitis". Current Health Sciences Journal 44.3 (2018): 280287.

28. "Corrigendum for Multiple Sclerosis: Associations Between Physical Disability and Depression Are Not Mediated by SelfReported Physical Activity". Percept Mot Skills (2019).

29. Abel S., et al. "Myelin Damage in Normal Appearing White Matter Contributes to Impaired Cognitive Processing Speed in Multiple Sclerosis". Journal of Neuroimaging 30.2 (2019): 205-211.

30. Jafarzadeh A., et al. "Vitamin D down-regulates the expression of some Th17 cell-related cytokines, key inflammatory chemokines, and chemokine receptors in experimental autoimmune encephalomyelitis". Nutritional Neuroscience 22.10 (2019): 725-737.

31. Lloyd AF., et al. "Central nervous system regeneration is driven by microglia necroptosis and repopulation". Nutritional Neuroscience 22.7 (2019): 1046-1052.
32. Lo Sasso B., et al. "Cerebrospinal Fluid Analysis in Multiple Sclerosis Diagnosis: An Update". Medicina (Kaunas) 55.6 (2019)

\section{Assets from publication with us}

- Prompt Acknowledgement after receiving the article

- Thorough Double blinded peer review

- Rapid Publication

- Issue of Publication Certificate

- High visibility of your Published work

Website: www.actascientific.com/

Submit Article: www.actascientific.com/submission.php Email us: editor@actascientific.com

Contact us: +919182824667 